\title{
NONLINEAR DAMPING FOR SHOCK PROTECTION OF MEMS DEVICES USING SHEAR THICKENING FLUID
}

\author{
S. Iyer ${ }^{1 *}$, H. Lee ${ }^{l}$, M. Liger ${ }^{2}, J . J u d y^{l}$ and R. Candler ${ }^{l}$ \\ ${ }^{1}$ Electrical Engineering Department, University of California, Los Angeles \\ ${ }^{2}$ Google, USA
}

\begin{abstract}
This manuscript reports the measurement and analysis of nonlinear viscous damping of MEMS actuators immersed in shear thickening fluids (STFs). The linear second-order system model is modified with a power-law damping term to account for the sheardependent viscosity of the fluid. This model is tested using a torsional microactuator immersed in a STF. At high actuation forces, the vibration velocity amplitude drops sharply. This result reveals the potential to use STFs as adaptive, passive dampers for shock protection of MEMS. To date, this is the only study that uses STFs in the context of dynamic damping for MEMS devices.
\end{abstract}

\section{INTRODUCTION}

Isolation from environmental disturbances is necessary for increased reliability and performance of micro-scale sensors and actuators for space, military and industrial applications. One of the most damaging external disruptions is mechanical shock caused by impact. Microscale devices with fragile mechanical structures have been shown to fracture as a result of large magnitude shocks [1]. Even when complete destruction of the device does not occur, shock can result in reduced performance and lifetime of MEMS devices.

Previous shock studies on MEMS devices focus on failure analysis [1], optimization of the mechanical structure design [1,2] or inclusion of an additional element for increased energy dissipation $[3,4]$. Adjusting device geometry to optimize for shock resistance creates a coupled design problem that results in a performance tradeoff with sensitivity. The inclusion of additional spring elements such as hard stops, soft wall coatings or nonlinear springs does not have this pitfall. However, these additional components do not prevent impact of the mechanical structure. In fact, the effectiveness of these structures for shock protection is limited because they can only dissipate energy when they are in direct, physical contact with the device structure.

Fluidic damping has the advantage that energy dissipation occurs continuously. For large-scale applications such as seismic protectors for buildings and automotive shock absorbers, dedicated, fluid-filled dampers can be used to absorb unwanted energy [5]. In micro-scale systems, however, it is impractical to have an isolated damping element. Instead, damping can be integrated into the device by encapsulating it in a viscous fluid [6]. The main drawback of this approach is that typical fluidic damping increases energy dissipation under all operating conditions, resulting in reduced performance.

In this work, we take advantage of shear thickening fluids (STFs) to create a passively damped system that adapts the damping force to the magnitude of the disturbing force. STFs have the unique property that their viscosity increases with increasing shear rate. Increased viscosity results in increased damping. Thus, STFs allow for normal device operation at low, working velocities, but severely restrict motion at high velocities typical of shocks. Here, we propose the use of STFs for increased energy dissipation in MEMS subject to mechanical shock.

\section{THEORY}

Studies on the mechanical dynamics of MEMS devices typically employ the use of a linear, second-order mass-springdamper model. Equation 1 is the equation of motion for such a system, where $x$ is displacement, $f(t)$ is the forcing function, $m$ is the device mass, $k$ is the spring constant and $b$ is the damping coefficient. The damping coefficient is determined by a number of energy dissipation mechanisms; however, in this case fluidic damping will be the dominant mechanism for energy loss.

$$
m \ddot{x}+b \dot{x}+k x=f(t)
$$

STFs are in a class of non-Newtonian fluids where viscosity is dependent on shear rate. The constitutive relation between viscosity and shear rate for these fluids is given in Equation 2,

$$
\eta=K \cdot \dot{\gamma}^{n-1}
$$

where $\eta$ is viscosity, $K$ is a material dependent constant, $\dot{\gamma}$ is shear rate and $n$ is the power-law index. For STFs, $n$ is greater than 1, so viscosity increases with shear rate. For Newtonian fluids, $n=1$ and viscosity is constant with shear rate. Finally, if $n$ is less than 1 , the fluid has shear thinning properties.

We can incorporate this nonlinearity in the second-order model by replacing the linear damping force that is proportional to velocity with a power-law damping term to account for increased damping under high amplitude actuation $[7,8]$. This substitution is shown in Equation 3. The absolute value and signum functions are

$$
m \ddot{x}+b|\dot{x}|^{n} \operatorname{sgn}(\dot{x})+k x=f(t)
$$

needed to fulfill the viscous damping requirement that the drag force always opposes the direction of motion. For a Newtonian fluid, the power-law index is equal to 1 . Equation 3 can then be simplified exactly to the linear ordinary differential equation presented in Equation 1. However, for shear thickening fluids, the power-law index $n$ is greater than 1. If we assume a sinusoidal input (as in Equation 4) with amplitude $A>0$, then the damping force will have a nonlinear dependence on both amplitude and drive frequency, $\omega$ (Equation 5). This will result in a damping force that has a greater than linear dependence on the magnitude of the actuation force. Additionally, we see that the sinusoid waveform will be distorted by the power-law dependence. Since typical power-law indices fall between 1 and 2, this distortion will result in the emergence of a $2 \omega$ frequency component in the damping force.

$$
\begin{aligned}
& x=A * \sin (\omega t) \\
& F_{d}=-b \cdot(A \omega)^{n}|\cos (\omega t)|^{n} \operatorname{sgn}[\cos (\omega t)]
\end{aligned}
$$

\section{METHODS \\ STF and Preparation}

Nearly all of the well-studied STFs consist of a colloidal suspension of particles in a solvent. Shear thickening results when the suspension transitions from a stable, ordered state to a state of flocculation, where motion causes small groups of particles to interact and form incompressible hydroclusters. Previous studies using STFs focus on characterization of the fluids alone or use them 
in isolated, macro-scale damping components $[9,5]$. This is the first study that employs the use of STFs as damping fluids for MEMS devices.

In this work, the STF used is a colloidal dispersion of 30\% (by weight) fumed silica nanoparticles (Aerosil 200 with $\sim 12 \mathrm{~nm}$ particle diameter) in a polyethylene glycol-200 (PEG) solvent. The silica nanoparticles are dispersed into the PEG solvent using a Thinky AR-100 conditioning mixer. Several hours of mixing are required to break up particle clusters and ensure an ordered dispersion is achieved.

\section{Torsional Device and Actuation}

The nonlinear damping effects of the STF were tested using a magnetically actuated torsional device [10]. The device and principle of actuation are shown in Fig. 1. In a uniform magnetic field, the magnet will try to align with the field, resulting in torsional motion.
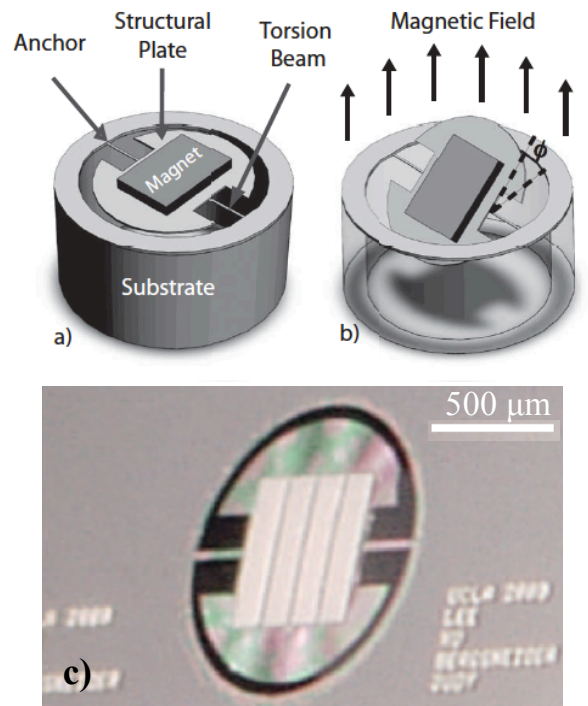

Figure 1: Torsional Si actuator: a) unactuated state, b) actuated by an applied magnetic field, c) released device.

The magnetic field needed to actuate the devices was produced using an external electromagnet. The torque produced by the electromagnet is given in Equation 6, where $M$ is the magnetism of the permanent magnet on the device, $V$ is the volume of the magnet and $H$ is the applied magnetic field strength. Equation 7 shows the expression for the magnetic field strength for a circular, air-core inductive coil, where $I$ is the current through the coil, $N$ is the number of turns, $a$ is the radius of the coil and $L$ is its length.

$$
\begin{gathered}
\tau_{\text {ext }}=(M \times H) \cdot V \\
H=-\frac{N \cdot I}{2 \sqrt{a^{2}+(L / 2)^{2}}}
\end{gathered}
$$

The electromagnet is driven using a custom-built transconductance amplifier from Marchand Electronics with a programmable input. The velocity of the device is then measured using a differential laser Doppler vibrometer. The complete experimental setup is shown in Fig. 2.

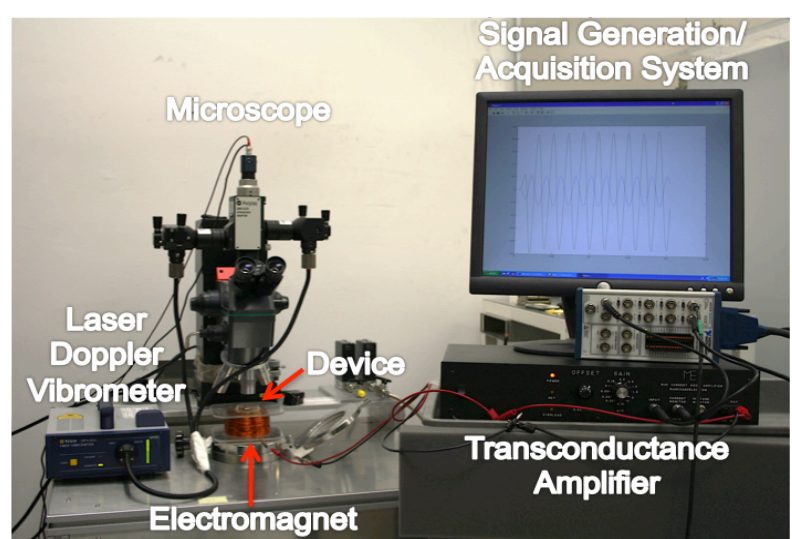

Figure 2: Experimental setup for measuring the velocity amplitude response. A laser Doppler vibrometer is used to measure the velocity of the device.

\section{RESULTS}

Typical studies on mechanical dynamics focus on the frequency response of a system. However, since we are trying to characterize the damping characteristics of the STF instead of the device performance, we are interested in the magnitude response of the damping force. Additionally, changes in the frequency response are difficult to identify and characterize since the system is highly damped.

In order to assess the damping properties of the fluid, we immersed the torsional actuator in the STF and measured the velocity amplitude as the magnitude of the sinusoidal actuation force was increased. The velocity amplitude response was also measured for two Newtonian fluids, PEG and air. Fig. 3 shows the response for a device immersed in STF versus PEG alone. Note that the two data series are plotted in the same velocity range to facilitate comparison of the respective damping forces in each fluid. In PEG, the actuator's velocity is quite linear with the magnetic actuation force, as we expect for a Newtonian fluid with linear viscous damping and power-law index $n$ equal to 1 . In the STF, however, the velocity does not increase linearly with drive force. At very high amplitude actuation, velocity drops of sharply, indicating that the motion of the device is limited despite a large excitation.

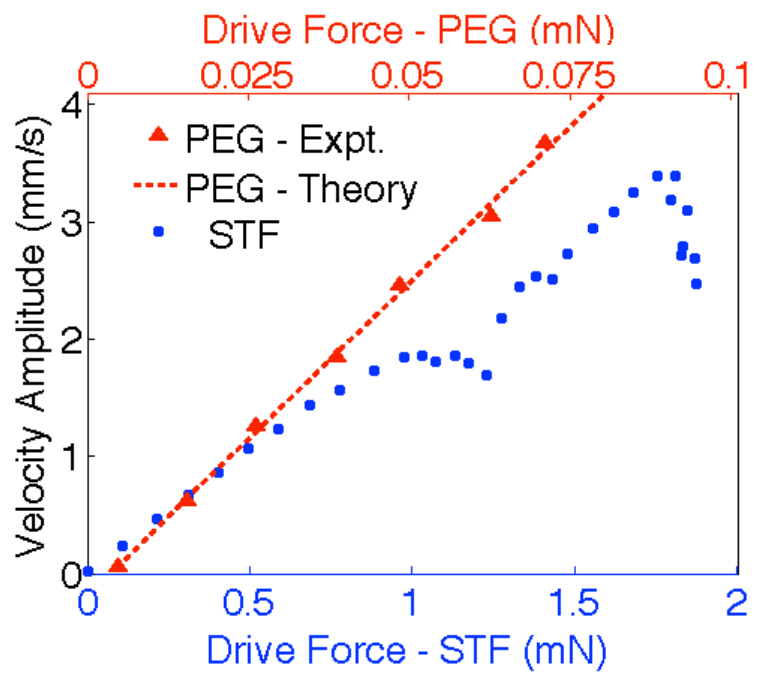

Figure 3: Velocity amplitude vs. actuation force for an actuator in PEG and STF. Two horizontal axes are used to match the velocity range of the device in each material. This facilitates a comparison of the damping, which depends on velocity. 
The Newtonian behavior of PEG alone is again verified in Fig. 4, which compares the velocity response in PEG with that in air, a fluid widely accepted as Newtonian. As expected, to achieve the same velocity in the more viscous PEG as in air, we require nearly twice the magnetic actuation force. The velocity responses in the Newtonian fluids are linearly dependent on actuation force, and we do not see the dramatic roll-off observed in the STF. This is a strong indication that the dispersion of fumed silica nanoparticles induces a shear thickening response in PEG.

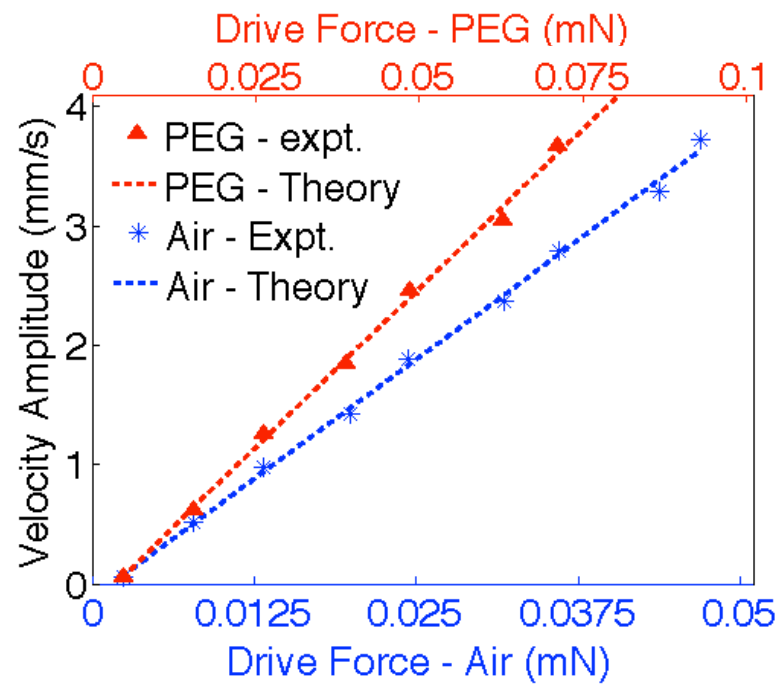

Figure 4: Velocity amplitude vs. actuation force amplitude for Newtonian fluids, PEG and air. The responses are similarly linear with theoretical fits corresponding to $n=1$ for both fluids. This suggests that the STF is responsible for the increased damping power.

The power-law damping model established in Equation 3 suggests that the shear-dependent viscosity of the STF results in a waveform distortion that manifests itself mathematically as an emergence of a frequency component at twice the drive frequency. Fig. 5 shows the relative contribution of the frequency components of the velocity for a torsional device immersed in the STF. Under low amplitude actuation, the $f_{\text {drive }}$ component is dominant.

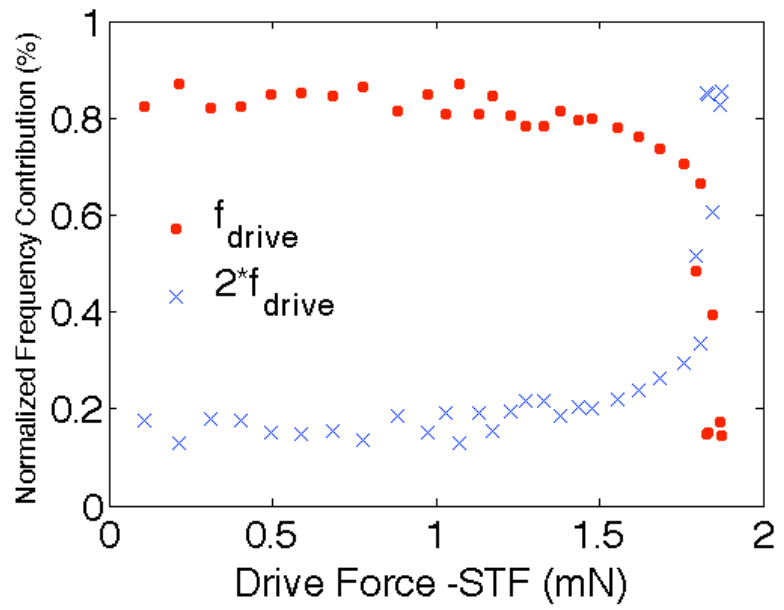

Figure 5: A plot showing the relative contribution of the first and second harmonics of the drive signal as actuation force is increased. In the shear thickening regime, the second harmonic becomes the dominant vibration mode.
However, at the onset of shear thickening behavior, there is a sharp decrease in the $f_{\text {drive }}$ component accompanied by an increase in the component at $2 * f_{\text {drive }}$. At high enough force amplitudes, the strength of the $2 * f_{\text {drive }}$ component surpasses that of the $f_{\text {drive }}$ component and becomes the dominant mode of vibration despite the fact that the forcing function is nearly a pure sinusoid with a single frequency. In fact, at high amplitude actuation forces, the device motion is primarily at twice the drive frequency. This is illustrated in Fig. 6, which superimposes the drive force and the velocity response in the time domain.

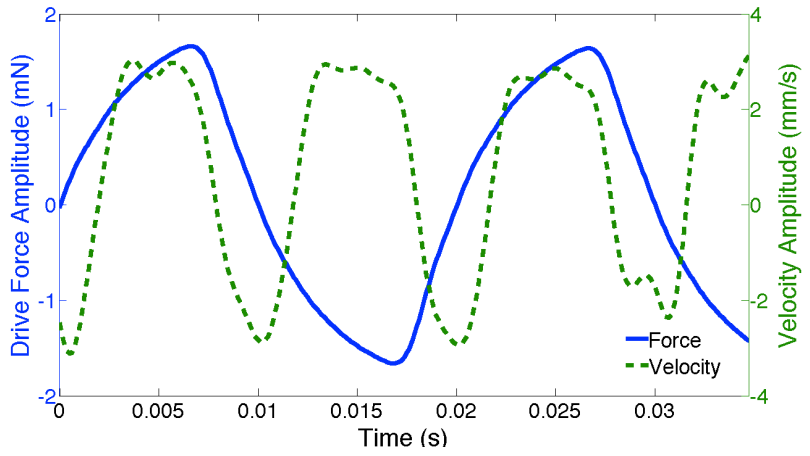

Figure 6: Time domain plots of the drive force and velocity under high amplitude actuation $(\mid$ Force $\mid=1.66 \mathrm{mN})$ at $50 \mathrm{~Hz}$. The velocity profile clearly shows oscillations at $100 \mathrm{~Hz}$.

\section{CONCLUSION}

We have successfully demonstrated a passively damped MEMS device where damping increases sharply when the device is subject to a high amplitude disturbing force. This damping effect is clearly a result of immersion in the STF and reveals the potential to use STFs as adaptive, passive dampers for shock protection of MEMS sensors or actuators. Additionally, we have shown that nonlinear damping due to the shear-dependent viscosity of the fluid can result in the emergence of a second vibrational mode at twice the drive frequency.

\section{ACKNOWLEDGEMENTS}

Student travel support has been generously provided by the Transducer Research Foundation.

\section{REFERENCES}

[1] D.M. Tanner, J.A. Walraven, K. Helgesen, L.W. Irwin, F. Brown, N.F. Smith, and N. Masters. "MEMS reliability in shock environments", Proceedings of the IEEE International Reliability Physics Symposium, San Jose, USA, April 10-3 (2000), pp. 129-138.

[2] J.K. Yee, H.H. Yang, and J.W. Judy. "Dynamic response and shock resistance of Ferromagnetic Micromechanical Magnetometers", Proceedings of the Tech. Digest IEEE Int. Conf. on Solid-State Sensors, Actuators, and Microsystems (TRANSDUCERS '03), Boston, USA (2003), pp. 308-311.

[3] S. Huang, X. Li, Y. Wang, J. Jiao, X. Ge, D. Lu, L. Che, K. Zhang, and B. Xiong. "A piezoresistive accelerometer with axially stressed tiny beams for both much increased sensitivity and much broadened frequency bandwidth", Proceedings of the Tech. Digest IEEE Int. Conf. on Solid-State Sensors, Actuators, and Microsystems (TRANSDUCERS '03), vol. 1, Boston, USA (2003), pp. 91-94 A.B. 
[4] S.W. Yoon, N. Yazdi, N. C. Perkins, and K. Najafi. "Micromachined integrated shock protection for MEMS", Sensors and Actuators A: Physical, Volumes 130-131, 14 August 2006, pp. 166-175.

[5] X.Z. Zhang, W. H. Li, and X. L. Gong. "The Rheology of Shear Thickening Fluid (STF) and the Dynamic Performance of an STF-filled Damper." Smart Materials and Structures 17.3 (2008).

[6] W. Zhang and K. Turner. "Frequency Dependent Fluid Damping of Micro/nano Flexural Resonators: Experiment, Model and Analysis." Sensors and Actuators A: Physical 134.2 (2007): 594-99.

[7] S. D. Dhole, R. P. Chhabra, and V. Eswaran. "Flow of PowerLaw Fluids Past a Sphere at Intermediate Reynolds Numbers." Industrial \& Engineering Chemistry Research 45.13 (2006): 4773-781.
[8] L.S. Jacobsen. "Steady forced vibrations as influenced by damping". Transactions ASME, 1930.

[9] F.J. Galindo-Rosales, F.j. Rubio-Hernández, and A. Sevilla. "An Apparent Viscosity Function for Shear Thickening Fluids." Journal of Non-Newtonian Fluid Mechanics (2011).

[10] H. Lee; S.A. Lee, M. Bergsneider, and J.W. Judy. "Unobstructing magnetic microactuators for implantable catheters." Solid-State Sensors, Actuators and Microsystems Conference (TRANSDUCERS 2009) ,vol., no., pp.857-860, 2125 June 2009

\section{CONTACT}

*S. Iyer, tel: +1-310-825-0585; ssiyer@ucla.edu 